\title{
ERS-1 Altimeter fast delivery data quality flagging over land surfaces
}

\author{
Fiona Strawbridge and Seymour Laxon \\ Mullard Space Science Laboratory, Department of Spece and Climate Physics, University College London
}

\begin{abstract}
Over land ice and land, satellite altimeters provide valuable topographic information, in spite of having been designed primarily to operate over the ocean. There is a need, however, for careful data quality assessment and screening as erroneous elevation measurements can be included within the telemetered data, especially when the range tracker encounters complex echoes, or rapidly varying topography. The ERS-1 Fast Delivery (FD) data product provides an excellent source of near real-time data, which has already been used for ice sheet mapping. This is a reduced data set consisting of onboard parameters generated once per second. In this paper we show that by applying thresholds to two parameters, significant amounts of poorly tracked data can be eliminated. The effectiveness of this filtering technique is demonstrated by a comparison of filtered and unfiltered altimeter data with a digital elevation model. This filtering technique is applied to the first 35 day repeat cycle of FD data obtained over land to produce the first map of global topography from ERS-1.
\end{abstract}

\section{Introduction}

As well as providing geophysical measurements over the ocean surface the ERS-1 altimeter was designed to map the topography of the ice sheets and the location of the sea ice boundary, and also produces useful measurements of elevation and surface roughness, over land and inland water surfaces [Guzkowska et al. 1990].

The altimeter transmits a radar pulse towards the surface, and records the echo in a series of power gates known as the "range window". To extract geophysical measurements from the echo, a leading edge, representing the first return from the surface, must be present within the range window [Martin et al. 1983]. Since the range window covers only a small fraction of the possible range variation around the satellite orbit, it is necessary to maintain the leading edge of the echo, or waveform, within the range window using an on-board tracker. ERS-1 can use one of two tracking modes. "Ocean mode" tracking uses a narrow range window for precise range measurement, whilst "ice mode" uses a range window four times wider to track surfaces with large range variations, such as ice sheets.

Under certain circumstances the echo shape is sufficiently distorted to confuse the on-board tracker such that it loses the leading edge of the return, and the altimeter is said to have "lost track". Loss of track will also occur where the topography varies too rapidly for the range tracker. Once this

\section{Copyright 1994 by the American Geophysical Union.}

Paper number 94GL01730

0094-8534/94/94GL-01730s03.00 occurs the telemetered echo and associated range measurements do not correspond to the surface. If useful geophysical information is to be extracted from altimeter data, data quality assessment is necessary, to screen out such data.

A reduced $1 \mathrm{~Hz}$ "Fast Delivery" data set is created by averaging the $20 \mathrm{~Hz}$ tracker estimates of range, Significant Waveheight (SWH) and Automatic Gain Control (AGC). This more compact data set is made available within hours of measurement. Although FD data are only generated when the instrument is in "ocean mode", they have already been used to map the topography of Greenland and Antarctica [Ridley et al., 1992; Tscherning et al., 1992].

Ideally data quality assessment should be carried out by analysing individual echoes. However these are not available in the FD data and an alternative method must therefore be used. This paper describes a procedure for assessing the quality of ERS-1 FD data over non-ocean surfaces.

\section{Data Quality Flagging}

Poor quality data, where the range window does not contain a leading edge, are often flagged as being in-track. This happens as the surface migrates out of the range window, but a delay occurs before the altimeter declares "loss of track" [Francis, 1990]. Users of altimeter data over the ocean interrogate a variety of parameters to flag bad data, including the Pulse Peakiness (PP) parameter, derived from the return pulse shape [Laxon and Rapley, 1987], SWH and AGC values [Brooks et al., 1990], and standard deviations of SWH, AGC and height [Hancock et al., 1980]. However, over non-ocean surfaces, the wide range and high spatial variability of surface height and reflectivity means that the usual tests will remove a large amount of valid data.

Scott et al. [1994] used an heuristic "dual gate" waveform analysis procedure over non-ocean surfaces to determine if a leading edge was present in the range window. Using this method we accept waveforms as valid only if the power in the last 44 bins exceeds $25 \%$ of that in bins 5 to 19 . Bins 1 to 4 are excluded since they contain aliased power [Francis, 1990].

Two elevation profiles (one in ocean-mode and one in icemode), covering the same ground track over part of the Antarctic ice sheet, are shown in figure 1 . The ice-mode tracker provided virtually continuous tracking of the surface (upper profile), whereas the ocean-mode tracker lost track periodically (lower profile). The dual gate waveform test was used to filter the ocean mode data, and the resulting elevation profile (middle profile), agrees well with the ice mode profile. This indicates that this waveform shape test provides an effective data quality flag. The absence of waveform data in the FD data set means that this waveform examination method can not be used. We will demonstrate that anomalously low 


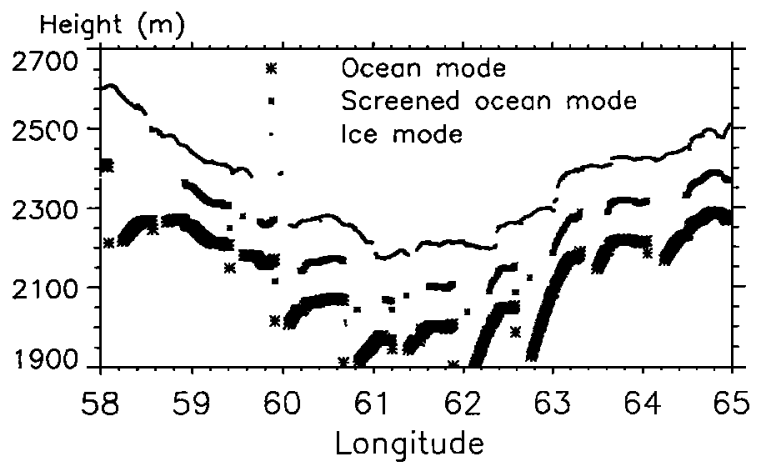

Fig. 1. Ice and ocean mode Antarctic height profiles. Ice mode data from 31/12/91, ocean mode data from 27/1/92.

values of PP can be used to flag data where a leading edge does not occur.

The standard deviations of altitude and SWH are larger over non-ocean surfaces due to real variations in the surface. SWH values are also frequently erroneous but the standard deviation of altitude does seem to be useful as a quality indicator. Use of the standard deviation of altitude is therefore also assessed.

The pulse peakiness parameter is defined by:

$$
\text { Pulse Peakiness (PP) }=\frac{31.5 \times \mathrm{P}_{\max }}{\sum_{\mathrm{i}=1}^{64} \mathrm{P}_{\mathrm{i}}}
$$

where $\mathrm{P}_{\max }$ is the maximum power in the range bins [Laxon \& Rapley 1987].

The behaviour of the pulse peakiness parameter during loss of track events over part of the Antarctic ice sheet is illustrated
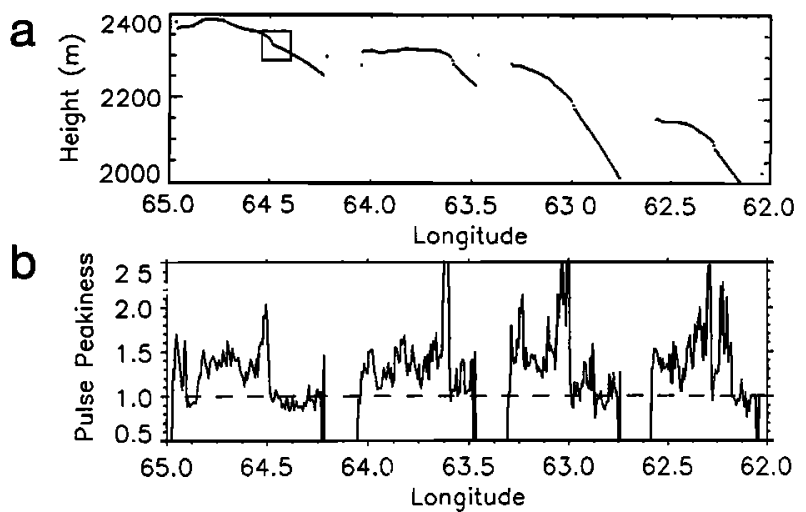

$$
\begin{aligned}
& P P=1.51 \text {, height=2353.4 } \\
& P P=11 \text {, height=2351.6 }
\end{aligned}
$$

Fig. 2a. Ocean mode Antarctic height profiles (27/1/92). Four loss-of-track events are shown. b) Corresponding PP values. c) Waveforms for the boxed loss-of-track event in a). The horizontal position of each range window with respect to the previous one indicates the relative location. Heights in $\mathrm{m}$. in figure 2. This figure shows a sequence of $20 \mathrm{~Hz}$ elevation and pulse peakiness measurements obtained on 27/1/92 over an area of ice sheet undulations in Antarctica. Several loss-oftrack events are clearly identifiable, where the elevation measurements drop rapidly after the surface has migrated out of the range window. The pulse peakiness value rapidly falls below 1 as the surface is lost, although the instrument continues in tracking mode for several seconds after the loss of track event. Waveforms for the first of these loss-of-track events (boxed in figure 2a) are shown in figure $2 c$. For the first seven waveforms the tracker manages to follow the downward slope of the topography well, with corresponding PP values of between 1.5 and 2.05; after waveform 8 the surface levels out, while the range window continues to shift downwards. This results in the waveform moving to the left of the range window, with a consequent drop in PP to values of $\sim 1$. By waveform 16, the leading edge has migrated out of the range window, and the subsequent waveforms are of poor quality. Corresponding PP values are generally $<1$, although occasional values of 1 or greater (e.g. waveform 19) are returned.

To evaluate the PP parameter as a data quality indicator both waveform and FD land data from 27/1/92 to 29/1/92 were analysed. Data with the standard deviation of altitude, $\sigma_{\text {alt }}$, set to zero (3329 points) were excluded as these indicate anomalous records. The remaining data set contained a total of 59944 data points. FD data records containing one or more waveforms which failed the dual gate test were classed as "bad", and the remainder as "good".

The histograms of pulse peakiness for good and bad quality data shown in figure 3 illustrate the trade-off between exclusion of bad data and retention of good data. The amounts of data included and excluded by different PP thresholds are shown in Table 1. These results show that a PP threshold of 1.0 will remove just $0.8 \%$ of the good data points, whilst excluding $34.1 \%$ of the bad data points. More bad data will be excluded by a slightly higher threshold, with a PP $=1.1$ threshold screening out $43.2 \%$ of bad data, with only $4.3 \%$ good data points being lost. Many non-ocean surfaces, such as ice sheets and desert areas, are characterised by ocean-like waveforms, which typically have PP in the range 1.2 to 1.5 . Thus adjustment of the threshold to greater than $\sim 1.1$ will exclude valuable data. On the basis of these results, a pulse peakiness threshold between 1 and 1.1 offers a reasonable compromise.

Filtering anomalous data by applying a threshold on the standard deviation of altitude $\left(\sigma_{\text {alt }}\right)$ is less straightforward as it will show high values both for loss of lock, and for rapidly

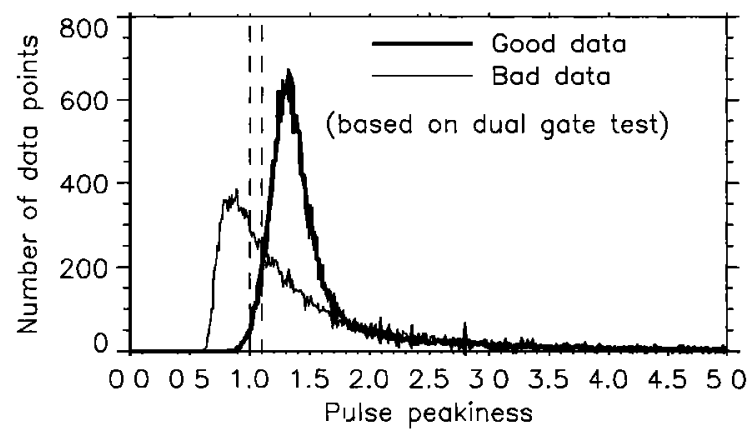

Fig. 3. Histograms of PP for data classified as "good" and "bad" using the dual gate test. Lines are shown at PP thresholds of 1.0 and 1.1. Global land data from 27-29/1/92. 
Table 1. Summary of the Effects of Different PP Thresholds upon the Quantities of Good and Bad Data Screened Out. Data to the left of the thresholds will be excluded (see figure 3). A total of 31448 data points were good and 28496 bad.

\begin{tabular}{ccccc}
\hline Peakiness & \multicolumn{2}{c}{ Good data excluded } & \multicolumn{2}{c}{ Bad data excluded } \\
Threshold & Number & $\%$ & Number & $\%$ \\
& & & & \\
\hline 0.9 & 21 & $0.1 \%$ & 6461 & $22.7 \%$ \\
0.95 & 91 & $0.3 \%$ & 8137 & $28.5 \%$ \\
1.0 & 265 & $0.8 \%$ & 9722 & $34.1 \%$ \\
1.05 & 655 & $2.1 \%$ & 11079 & $38.8 \%$ \\
1.1 & 1360 & $4.3 \%$ & 12323 & $43.2 \%$ \\
1.15 & 2572 & $8.2 \%$ & 13425 & $47.1 \%$ \\
1.2 & 4391 & $14.0 \%$ & 14466 & $50.7 \%$ \\
\hline
\end{tabular}

varying topography. Histograms of $\sigma_{\text {alt }}$ for good and bad data, after application of the $P P \geq 1$. 1 filter, are shown in figure 4. The effects of choosing different $\sigma_{\text {alt }}$ thresholds are evaluated in Table 2 . In contrast to the PP thresholds, where data falling to the left of the threshold are rejected, for $\sigma_{\text {alt }}$, data to the right of a given threshold will be rejected. A $\sigma_{\text {alt }}$ threshold of $20 \mathrm{~m}$ will exclude just $2.6 \%$ of the total good points, while removing $22.8 \%$ of the bad points. Choice of a lower $10 \mathrm{~m}$ threshold will exclude more bad data $(48.3 \%)$, but will exclude more good data points (7.1\%), particularly in regions characterised by large surface slopes which may be of interest, such as the ice sheet margins.

The geographical distribution of data points that are excluded using three $\sigma_{\text {alt }}$ thresholds $(5,10$ and $15 \mathrm{~m}$ ) together with the PP = 1 threshold, was compared with the distribution of data removed using the dual gate waveform shape test, for the three day repeat of data over Western Antarctica. Visual comparison revealed a very good correspondence between the location of data retained by the dual gate test and those retained using PP $\geq 1, \sigma_{\text {alt }} \leq 15 \mathrm{~m}$. Use of lower $\sigma_{\text {alt }}$ thresholds often resulted in long sequences of good data being screened out.

These results suggest that the $\sigma_{\text {alt }}$ parameter can contribute to data quality flagging for FD data, and that a high $\sigma_{\text {alt }}$ threshold, of the order of $15 \mathrm{~m}$, will remove a substantial amount of bad data $(\sim 33 \%)$ whilst retaining a large number of good quality returns $(\sim 96 \%)$ over well tracked areas. Of our 59944 point data set, using the combination of $P P \geq 1.1$ and $0>$ $\sigma_{\text {alt }} \leq 15 \mathrm{~m}$, a total of $28895(48.2 \%)$ data points were of good quality and accepted, 2553 (4.3\%) were good but rejected, $17653(29.4 \%)$ were bad and rejected, and 10843 (18.1\%) were bad but accepted.

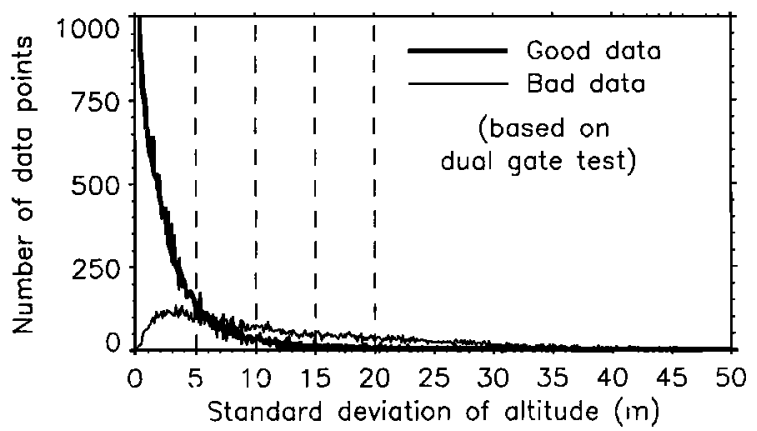

Fig. 4. Histograms of $\sigma_{a l t}$ for data classified as "good" and "bad" using the dual gate test, which have already been screened for $P P \geq 1.1$. Lines are shown at $\sigma_{\text {alt }}$ thresholds of $5 \mathrm{~m}, 10 \mathrm{~m}, 15 \mathrm{~m}$ and $20 \mathrm{~m}$. Global land data from 27-29/1/92.
Table 2. Summary of the Effects of Different $\sigma$ alt Thresholds upon the Proportions of Good and Bad Data Screened Out. For data which have already had the $P P \geq 1.1$ screen applied. Data to the right of the thresholds will be excluded (see figure 4). A total of 30088 data points were good, and $16173 \mathrm{bad}$.

\begin{tabular}{ccccc}
\hline Oalt & \multicolumn{2}{c}{ Good data excluded } & \multicolumn{2}{c}{ Bad data excluded } \\
Threshold & Number & $\%$ & Number & $\%$ \\
& & & & \\
\hline 6 & 4111 & $13.7 \%$ & 10638 & $65.8 \%$ \\
8 & 2872 & $9.5 \%$ & 9055 & $56.0 \%$ \\
10 & 2151 & $7.1 \%$ & 7808 & $48.3 \%$ \\
12 & 1642 & $5.5 \%$ & 6663 & $41.2 \%$ \\
14 & 1330 & $4.4 \%$ & 5766 & $35.7 \%$ \\
15 & 1193 & $4.0 \%$ & 5330 & $33.0 \%$ \\
16 & 1091 & $3.6 \%$ & 4935 & $30.5 \%$ \\
18 & 917 & $3.0 \%$ & 4251 & $26.3 \%$ \\
20 & 768 & $2.6 \%$ & 3683 & $22.8 \%$ \\
\hline
\end{tabular}

\section{Topographic mapping using Fast Delivery data}

The data quality assessment procedures described above were used to produce the map of global topography shown in figure 5. This map was compared with the ETOP05 global digital elevation model (DEM).

Fast Delivery altimeter data from a 35-day repeat (15/8/92 to $18 / 9 / 92$ ) were mapped to the same 5 arc-minute grid as ETOP05. When the ETOP05 heights were subtracted from global altimeter measurements, gross and systematic errors were observed in some regions, resulting in part from the use of different datum's in compiling the ETOP05 model. Thus many of the discrepancies between the data sets are due to deficiencies in the DEM [Wingham et al. 1992]. However the DEM is known to be more accurate in Europe, the USA, Japan and Australia, and we use data over Australia for a more detailed study.

The ETOP05 elevation measurements were subtracted from corresponding altimeter measurements, for unfiltered altimeter data and for two levels of filtered altimeter data. The filtered altimeter data sets were created using $P P \geq 1.1$, together with $\sigma_{\mathrm{alt}} \leq 15 \mathrm{~m}$ for one data set, and a stricter $\sigma_{\mathrm{alt}}$ filter of $5 \mathrm{~m}$ for the second. Histograms of the differences between the filtered altimeter data and ETOP05 are narrower and less skewed than the differences observed with the raw data (figure 6). As expected, a significant amount of altimeter data with elevations below ETOP05, resulting from the downward migration of the range window after loss of track, are eliminated in the filtered data. The narrowest histogram is obtained using the very strict $\sigma_{\text {alt }} \leq 5 \mathrm{~m}$ filter; however this $\sigma_{\text {alt }}$ filter also removes more data which agree well with ETOP05.

\section{Conclusions}

In this paper we have investigated the usefulness of the Pulse Peakiness parameter (PP) and standard deviation of altitude $\left(\sigma_{\text {alt }}\right.$ ) for assessing the quality of ERS-1 FD data. The normal data quality criteria used over the ocean are unsuitable for land data where the surface varies more rapidly. By comparing values of FD parameters with corresponding echo data we have been able to determine their utility for data quality assessment. We have shown that $\mathrm{PP}$ is extremely useful for eliminating data on occasions where the altimeter has lost lock. 


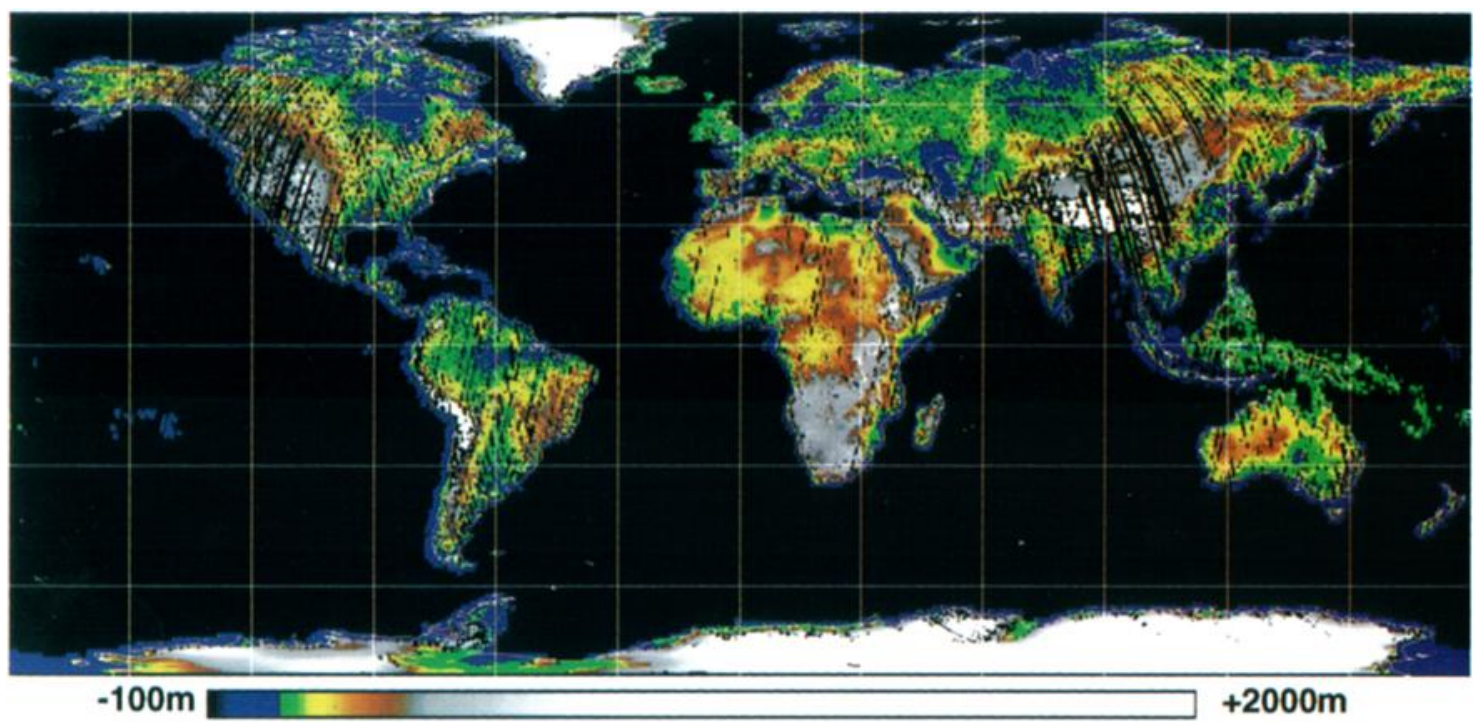

Fig. 5. Global land topography from a 35 day repeat of filtered FD data. Heights in metres above the reference ellipsoid.

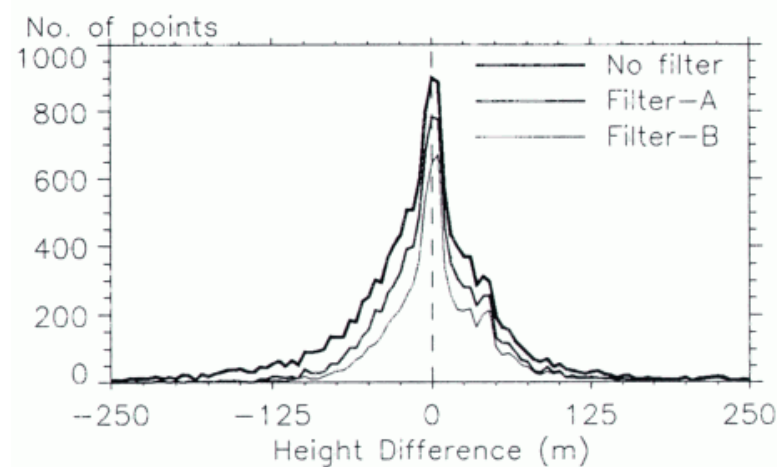

Fig. 6. Histograms of altimeter - ETOP05 elevation differences over Australia, for both filtered and unfiltered altimeter data. For (A) the $P P \geq 1.1$ and oalt $\leq 15 \mathrm{~m}$ filters were used; for (B) the stricter $\sigma$ alt $\leq 5 \mathrm{~m}$ filter was added. Fast Delivery 35 day repeat data from 15/8/92-18/9/92.

Up to $43 \%$ of poorly tracked data can be eliminated using a simple threshold on PP (1.1) with loss of good data of only $4.3 \%$. The use of $\sigma_{\text {alt }}$ is more problematic since this can be high in areas of large surface slopes, as well as where the altimeter has lost lock. In this case a trade-off must be made between elimination of poor data and retention of good data.

Using a threshold of 1 on the PP parameter and $15 \mathrm{~m}$ on $\sigma_{\text {alt }}$ we have generated the first global topographic map from the ERS-1 altimeter. This data set provides the most dense global coverage yet gathered over the land, and maps areas above $72^{\circ}$ for the first time.

Acknowledgements. This work was in part funded under contract RAE 1B/44 of the Defence Research Agency, as a subcontract of ESA contract 9575/91/HGE-I. We are grateful to Helen Snaith and Trevor Guymer of the James Rennell Centre for their careful review.

\section{References}

Brooks, R., D. Lockwood \& D. Hancock, Effects of islands in the Geosat footprint, I. Geophys. Res., 95, c3, pp. 2849-2855, 1990.

Francis, C., RA data chains, ESA document no. ER-IS-ESAGS-0001, 1990.

Guzkowska, M., C. Rapley, J. Ridley, W. Cudlip, .C. Birkett, \& R. Scott, Developments in Inland Water and Land Altimetry, ESA CR-7839/88/F/FL, 1990.

Hancock, D., R. Forsythe \& J. Lorell, Seasat altimeter sensor file algorithms, IEEE J. Oc. Eng, 5, 2, pp. 93-99, 1980.

Laxon, S. \& C. Rapley, Radar altimeter data quality flagging, Adv, in Space Res., 7 ,(11), pp. 315-318, 1987.

Martin, T., A. Brenner, H. Zwally \& R. Bindschadler, Analysis and retracking of continental ice sheet radar altimeter waveforms, J.Geophys. Res., 88, 1983.

Ridley, J., S. Laxon, C. Rapley \& D. Mantripp, Antarctic ice sheet topography mapped with the ERS-1 radar altimeter, Int.J.Rem. Sens 14, 9, pp.1649-1650, 1993.

Scott, R., S. Baker, C. Birkett, S. Laxon, D. Mantripp, J. Mansley, J. Morley, C. Rapley, J. Ridley, F. Strawbridge \& D. Wingham. A comparison of the performance of the ice and ocean tracking modes of the ERS-1 radar altimeter over non-ocean surfaces, In press Geophys. Res. Letts. 1994.

Tscherning, C., P. Knudsen, S. Ekholm \& O. Baltazar Andersen, An analysis of the gravity field in the Norwegian Sea and mapping the ice cap of Greenland using ERS-1 altimeter measurements, paper presented at Cannes ERS-1 PI meeting, 1992.

Wingham, D., C. Birkett, R. Devayya, L Gilbert, D. Jackson, D. Palmer, C. Rapley. \& M. Wolf, Global digital elevation model assessment, MSSL Report 4030/10-92/001, UCLDRA Agreement no:2047/144/EXR3 1991, 1992.

F. Strawbridge and S. Laxon, Mullard Space Science Laboratory, University College London, Holmbury St. Mary, Dorking, Surrey RH5 6NT, United Kingdom.

(Received March 22, 1993; revised June 23, 1993; accepted February 25, 1994) 